\title{
Towards a physiological signal-based access solution for a non-verbal adolescent with severe and multiple disabilities
}

Negar Memarian, Stefanie Blain-Moraes \& Tom Chau

To cite this article: Negar Memarian, Stefanie Blain-Moraes \& Tom Chau (2014) Towards a physiological signal-based access solution for a non-verbal adolescent with severe and multiple disabilities, Developmental Neurorehabilitation, 17:4, 270-277, DOI: 10.3109/17518423.2013.834997

To link to this article: https://doi.org/10.3109/17518423.2013.834997

曲 Published online: 02 Oct 2013.

Submit your article to this journal $\pi$

Џ Article views: 136

Q View related articles 두

View Crossmark data

Citing articles: 2 View citing articles $\asymp$ 


\title{
Towards a physiological signal-based access solution for a non-verbal adolescent with severe and multiple disabilities
}

\author{
Negar Memarian", Stefanie Blain-Moraes², \& Tom Chau ${ }^{3,4}$ \\ ${ }^{1}$ David Geffen School of Medicine, University of California Los Angeles, Los Angeles, CA, USA, ${ }^{2}$ Department of Anaesthesiology, \\ University of Michigan, Ann Arbor, MI, USA, ${ }^{3}$ Bloorview Research Institute, Holland-Bloorview Kids Rehabilitation Hospital, Toronto, ON, \\ Canada, and ${ }^{4}$ Institute of Biomaterials and Biomedical Engineering, University of Toronto, Toronto, ON, Canada
}

\section{Abstract}

Objective: To find physiologically arousing stimuli and labile physiological channels in a nonverbal adolescent with severe and multiple congenital disabilities, who did not have a reliable means of communication.

Methods: The client was repeatedly presented with visual and audiovisual stimuli, representing variations of six contextual factors over three sessions in a one month period. For each stimulus, reactions were detected in the client's four peripheral autonomic nervous system signals using a rule-based classification algorithm.

Results: During the presentation of audiovisual stimuli, the number of physiological reactions significantly differed from that observed in baseline $\left(\chi^{2}=3.93, p=0.0476\right)$. Aural stimuli articulated in an unfamiliar voice, and aural stimuli containing anticipatory patterns were also physiologically arousing. Fingertip temperature was the client's most labile physiological signal. Conclusions: The results of this case study suggest that physiological data may complement caregiver acumen in deciphering the reactions of non-verbal clients with severe and multiple disabilities.
\end{abstract}

\section{Keywords}

Access pathway, arousal, people with severe disabilities, physiological signals

\section{History}

Received 9 August 2013

Revised 12 August 2013

Accepted 12 August 2013

Published online 1 October 2013

\section{Introduction}

\section{The communication challenge}

People with severe and multiple disabilities are often aware of events and stimuli in their immediate environment, but as a result of their limited physical abilities, are unable to interact with their surroundings through consistent and meaningful expressions or movements [1]. These individuals may therefore appear completely passive; they do not have the ability to deliberately and voluntarily convey their intentions, but rather rely completely on others (e.g. parent, nurse, or caregiver) to discern and interpret their needs. The inability to physically express personal intentions may be secondary to neurological conditions such as cerebral palsy, degenerative neuromuscular disorders such as muscular dystrophies and amyotrophic lateral sclerosis, autoimmune diseases such as multiple sclerosis, and strokes, especially in the brain stem [2]. In order to provide this population with some degree of independence, design, and development of access pathways has become an important topic in rehabilitation research.

Correspondence: Tom Chau, Bloorview Research Institute, Holland Bloorview Kids Rehabilitation Hospital, 150 Kilgour Road, Toronto, Ontario M4G 1R8, Canada. Tel: 416-425-6220, extension 3515. E-mail: tom.chau@utoronto.ca
An access pathway is the actual sensors or input devices by which an expression of functional intent (e.g. a movement or physiological change) is transduced into an electrical signal [3]. In other words, it is the conduit which connects an assistive technology, such as augmentative and alternative communication, to its user. Unfortunately, most of the commercially available alternative access pathways (e.g. mechanical switches, eye gaze systems, and inertial sensors) require at least one repeatable movement [3], and hence for many individuals, cannot help to resolve this communication challenge.

\section{An alternative communication solution}

While many people with severe and multiple disabilities often do not have the ability to voluntarily control their skeletal muscular system, their sympathetic sudomotor function may remain intact [4]. The individual's sympathetic activity is manifested in the central and peripheral signals of the autonomic nervous system (ANS). Signals of the peripheral ANS are modulated by affective and startling stimuli [5-8], and have been used to monitor human emotion [9-12]. An emerging physiological signal-based access pathway for people with severe disabilities is one that works based on voluntary control of peripheral ANS signals. It has been shown that by performing mental activities such as music imagery or mental arithmetic, one can voluntarily generate reactions in his/her peripheral ANS signals $[13,14]$. 


\section{Motivation and objectives}

This paper represents a foundational step towards the development of a communication solution for a non-verbal adolescent with severe and multiple congenital disabilities [15]. The first step in developing a physiological access pathway is the identification of stimuli that elicit meaningful and reproducible changes in an individual's physiological signals. Biofeedback monitoring may provide an objective way of detecting change-inducing stimuli $[9,11]$. Thus, the primary objective of this study was to identify stimuli that cause significant reaction in the client's physiological signals. This purpose is analogous to stimulus preference screening in case studies with microswitches [16-18]. Lancioni et al. [19] defined preferred stimuli as those that that seemed to produce positive reactions (e.g. alert or smiling) by the client. We emphasize that in the context of the present case study, preferred (arousing) stimuli are stimuli that elicit meaningful and reproducible change in the client's physiological signals.

The secondary objective of this study was to identify the client's most labile physiological channels (i.e. physiological signals that contain the greatest number of reactions to arousing stimuli). Addressing these objectives will help to answer the question about whether or not physiological signals can be used to determine suitable stimuli for a potential physiological access solution for an individual with severe disability.

\section{Materials and methods}

\section{Peripheral ANS signals}

We used four peripheral ANS signals (electrodermal activity (EDA), fingertip temperature, heart rate, and respiration) as a means of identifying arousing stimuli. EDA is a measure of the sympathetic cholinergic function that induces changes in the skin's resistance to electrical conduction. Changes in EDA can be spontaneously or reflexively evoked by a variety of internal or external arousal stimuli [20]. These stimuli cause an increase in EDA of more than $0.05 \mu \mathrm{S}$ within a 10 -s interval - a characteristic change known as an electrodermal reaction (EDR) [21]. These reactions occur with a latency of 1.3-1.65 s subsequent to stimulus presentation for persons without physical disabilities and between 1.5 and $2 \mathrm{~s}$ for subjects with disability such as cerebral palsy [4, 22]. Recently, these electrodermal signals have demonstrated potential as an access pathway [13].

Fingertip temperature fluctuations are a combination of long-term changes due to homeostatic thermoregulation, and transient changes in cutaneous microcirculation [23]. These transient changes are often a result of the contraction and dilation of arteriovenous anastomoses (AVAs), which are densely innervated by sympathetic nerve fibres, and hence reflect the state of an individual's ANS [23]. The direction of these changes is dependent on the overall body temperature the same stimuli can elicit both vasoconstriction and vasodilation [24].

The effects of startling or arousing stimuli on human cardiac signals have been often reported in the literature. For example, Lacey and Lacey [25] showed that infrequently occurring aural tones embedded in a sequence of standard auditory stimuli generated bradycardia, or slowed heart rate. In a separate study, an acute loud noise was found to induce increases in blood pressure and heart rate, likely due to sympathetic activation [5].

When in a relaxed state, an individual's respiration rate and amplitude maintain a steady-state pattern; these features change when the individual experiences stress [26]. Generally, respiratory responses tend to vary with the levels of valence and arousal of affective stimuli [27]. Furthermore, Jonghwa and Andre [28] found that features in both electrocardiographic and respiratory signals were useful for emotional differentiation.

\section{Case description}

The client, whom we will refer to as Sean, was a 15-year-old male with spastic quadriplegic cerebral palsy, visual impairment, and global developmental delay, secondary to birth asphyxia. He had elevated or fluctuating tone throughout his extremities, and reduced or fluctuating tone in his neck and trunk. Sean's personalized equipment included bilateral wrist splints and ankle-foot orthoses and a Tilt-in-Space manual wheelchair. Sean lived at home with his family and attended a special education school. He had a history of infantile seizures, ongoing respiratory and swallowing difficulties, gastroesophageal reflux secondary to hiatus hernia and oesophageal stricture, and recurrent bronchiolitis and pneumonia. He also had a sleep disorder characterized by significant sleep apnea due to airway obstruction and poor onset of sleep. Sean depended on trained caregivers for all activities of daily living and was enterally fed via a gastrostomy tube. His primary caregiver was his mother, who reported an ability to discern preference based on facial expression, body language, and an idiosyncratic yes/no response (i.e. opening his mouth for yes, and pursing his lips for no). This response was not consistent, and usually required the interpretation of a familiar caregiver. Over the years, Sean had tried numerous assistive devices to facilitate communication, including a Big Mac switch mounted above Sean's left arm, a chin switch and lever switch. As well, different communication strategies had been entertained, including auditory scanning, a picture communication book and various switch-activated software programs. At the time of the study, these aforementioned devices and strategies had all been abandoned, and Sean's preferences and responses were primarily interpreted by the attending caregiver.

\section{Instrumentation}

The aforementioned peripheral ANS signals were noninvasively acquired using a ProComp Infiniti multi-modality encoder from Thought Technology Ltd. and a laptop computer. EDA was measured via two $\mathrm{Ag}-\mathrm{AgCl}$ electrodes (SA9309M, Thought Technology Ltd.) placed on the palmar surface of the second and third finger of Sean's left hand. Heart rate was recorded using a photoplethysmography sensor (SA9308M, Thought Technology Ltd.) fastened to the tip of his fourth finger. Thoracic respiration was measured using a stretch sensitive belt (SA9311M, Thought Technology Ltd.) positioned around the upper aspect of the trunk. Skin temperature was detected with a 0.125 in. bead thermistor 
Figure 1. Instrumentation set-up for acquiring the peripheral ANS signals in the present study (EDA; BVP = blood volume pulse).

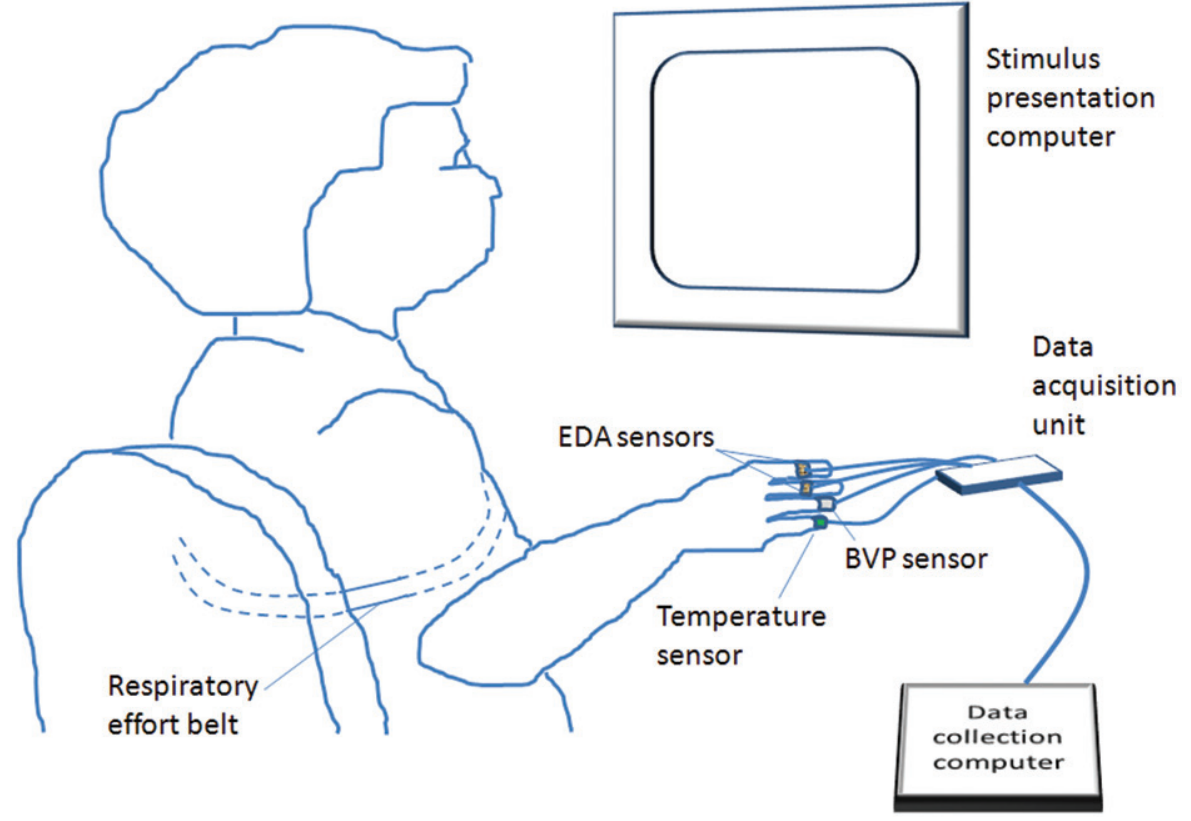

(SA9310M, Thought Technology Ltd.) securely fastened to the fifth finger of Sean's left hand. Figure 1 summarizes the instrumentation set-up.

\section{Study protocol}

Three data collection sessions were conducted over three different days within a one month period, in a controlled, indoor hospital environment with minimal distraction. Any unexpected events such as alerts from the Physical Address (PA) system, client coughing, sneezing, or extraneous movement were recorded by the researchers to help filter the data during the subsequent offline analysis. In each session, Sean was seated in his wheelchair, facing a computer screen. At the beginning of each session, a baseline recording of at least $300 \mathrm{~s}$ was obtained, wherein no stimulus was presented. This was followed by several stimulus presentations, as described in Table I. We consulted Sean's primary caregiver (his mother) to identify these candidate stimuli. The stimuli were presented on a laptop computer, which was connected to two speakers (for presenting the audio stimuli). Over the three sessions, Sean completed each stimulus test at least twice. No prior information about the stimuli was given to him. Since the purpose of this study was to examine Sean's natural reactions as manifested in his physiological signals, the researchers and his parent refrained from talking to him during the presentation of stimuli. Also, in order to prevent distraction during the tests, the researchers and the parent stayed out of Sean's sight, such that only the laptop, which presented the stimuli was in his field of view. The protocol was approved by the research ethics board of the paediatric rehabilitation hospital and affiliated university. Sean's mother provided written consent for participation in this study.

\section{Data analysis}

The recorded physiological data were analysed offline. Initially, each physiological signal was visually inspected for potential reactions, i.e. significant deviations from baseline values or patterns. To quantitatively detect these reactions, the following algorithms were employed.

EDRs, indicators of potential sympathetic nervous responses, were detected using the algorithm presented by Blain et al. [13], which tracks the centroid of the first derivative of the EDA signal. The total number of EDRs per trial was tabulated.

Significant changes in the slope of the temperature in either a positive or a negative direction were tracked. A leastsquares line was fit to the first $5 \mathrm{~s}$ of data. The cumulative mean square error from this line was then computed at 1-s intervals. A significant slope change was identified when the cumulative mean square error exceeded an empirically determined threshold. At this point, a new least-squares line was estimated with the next $5 \mathrm{~s}$ of data and the above process iterates. The total number of significant changes in slope was tabulated for each trial.

The respiration rate and amplitude were calculated over 5-s windows for the baseline trial of each session with consecutive windows overlapping by $1 \mathrm{~s}$. In the two-dimensional feature space defined by respiration rate and amplitude, we identified the centre of the cluster (mean vector) and assumed a radially symmetric distribution. The contour which enveloped $95 \%$ of the baseline data was defined as the boundary of the baseline state. For each of the subsequent trials recorded during that session, respiration rate and amplitude of each second of data recording were extracted, forming a trajectory in the two-dimensional feature space. Points that fell outside the boundary of the baseline state were indicative of a change in respiration. The total number of such changes was summed over the duration of each trial.

The inter-beat interval (IBI) of Sean's heart was calculated from the raw blood volume pulse data collected during each trial. Taking the inverse of the IBI signal yielded an estimate of Sean's heart rate. For the duration of the trial, changes in heart rate were automatically scored, such that an increase in heart rate (i.e. acceleration) yielded a score of +1 , and a decrease in heart rate (i.e. deceleration) yielded a score of -1 . 
Table I. Stimuli investigated in reaction tests with the client.

Factor Variations

(1) Presentation modality $\quad$ (a) Visual

(b) Audiovisual

(2) Familiarity with stimulus

(a) Content

(b) Tone

(3) Colour

(4) Startle

(5) Anticipation

Red, blue, green, yellow, and black

(a) Random sounds-paper crumple

(b) Waves and glass breaking

(a) Drum roll-ta-da

(b) Knock-chime

(6) Association

(a) Talk-clap

(b) Talk-music
Detail

A random sequence of two types of visual stimuli: (1) images of the planets in the solar system and (2) picture of persons very familiar to the client (his mother and sister). Duration: $100 \mathrm{~s}$

A random sequence of two types of visual stimuli: (1) fireworks with sound and (2) grey static screen without sound. Duration: $80 \mathrm{~s}$

A random audio sequence of words unfamiliar to the client (name of different planets in the solar system) randomly interrupted with one familiar word (client's name). Duration $100 \mathrm{~s}$

Repetition of experiment 2a with words spoken in familiar (maternal) and unfamiliar voices. Duration: $100 \mathrm{~s}$

Picture of a toy car moving in random directions on the display. Every $5 \mathrm{~s}$, the car picture colour changed. Duration: $100 \mathrm{~s}$

A random sequence of dull repetitious sounds (e.g. hammering and knocking) and paper crumple (startle stimulus identified by client's parent). Duration: $100 \mathrm{~s}$

A random sound sequence of a shoreline waves (soft and relaxing sound) and glass breaking (sharp and startling sound). Duration: $80 \mathrm{~s}$

An auditory pattern consisting of a drum roll followed immediately by a ta-da alert. After several repetitions the drum roll was followed by silence. Duration: $55 \mathrm{~s}$

An auditory pattern consisting of knocking followed immediately by a high pitch chime. After several repetitions, the knocking was followed by silence; duration $55 \mathrm{~s}$

An auditory pattern consisting of a lady's voice saying 'one, two, three, clap!' followed immediately by the sound of applause. After several repetitions, the speech was followed by silence; duration $58 \mathrm{~s}$

An auditory pattern consisting of a man's voice saying '[Client's name], let's play music!' followed immediately by music. After several repetitions, the speech was followed by silence; duration $85 \mathrm{~s}$
The net change in heart rate (sum of the indicator sequence) and the local maximum heart rate were estimated over consecutive 5-s windows where neighbouring windows overlapped by $1 \mathrm{~s}$. The resulting points populated a twodimensional feature space defined by the net change in heart rate and the local maximum heart rate. The points generated during baseline were assumed to have originated from the same physiologic state; the centroid of all points was calculated and a radially symmetric joint distribution was assumed. The contour which encompassed $95 \%$ of the points was defined as the boundary of the baseline state. The same two features (i.e. net change in heart rate and local maximum heart rate) were extracted for each second of each subsequent trial. Those that fell outside of the boundaries of the baseline cluster were flagged as changes in cardiac activity. The total number of such changes was summed over each trial.

Since all of the above analyses proceeded in one segment increments, there was at most one reaction per second per signal modality. For each trial, we counted the number of reactions, $N$, among the four physiological signals. Equivalently, this quantity could be interpreted as the total time in seconds where Sean was responsive. For each condition in Table I, the tally, $N$, of reactions was compared with the total number of physiological reactions during baseline via a $\chi^{2}$ test of homogeneity of proportions [29] at a $5 \%$ significance level.

\section{Results}

Figure 2 shows an example of Sean's baseline EDA, heart rate, temperature, and respiration signals. Note the monotonic behaviour of the EDA and temperature recordings, and the periodic pattern of respiration and blood volume pulse signals. The dip in respiration amplitude at about $60 \mathrm{~s}$ is likely due to a shift in Sean's trunk position, which in turn induced a sudden change in the average tension of the respiration band.

Table II summarizes the number of reactions during the stimulus trials and their comparison with baseline reactions. For this comparison, we included all the baseline recordings from Sean, resulting in a total of $2331 \mathrm{~s}$ of recording time and 520 reactions. The cells marked with asterisks represent trials where the total number of physiological reactions significantly differed from that observed in the corresponding sessional baseline $\left(\chi^{2}\right.$ test, $\left.p<0.05\right)$.

Table III presents the distribution of detected reactions across the four physiological signals within each type of trial. In all trials except one (anticipated aural pattern), Sean's temperature signal was his most labile physiological modality, implying that he is a temperature responder. Between the four physiological signals, respiration amplitude demonstrated the smallest proportion of reactions. Given that Sean had ongoing respiratory difficulties and a history of pneumonia, respiratory lability may have been compromised.

\section{Discussion}

The stimuli used in this study were selected at the discretion of Sean's mother, who was his primary caregiver. Our results include instances of agreement between caregiver predictions and reactions in Sean's physiological signals. Figure 3 depicts sample recordings from the presentation modality test 

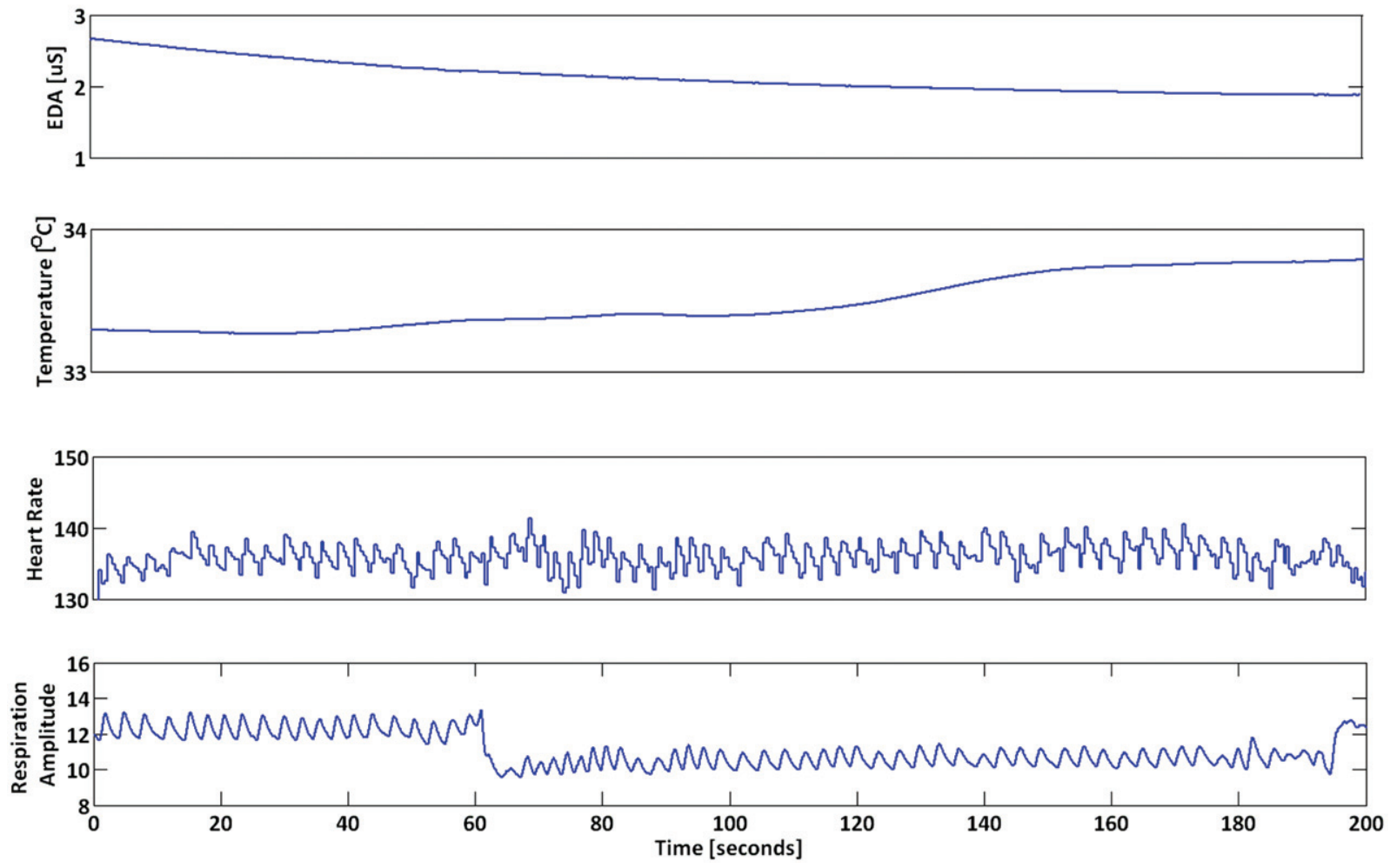

Figure 2. Client's physiological signals in relaxed state (baseline); from top to bottom: EDA, fingertip temperature, heart rate, and respiration amplitude. Heart rate and respiration amplitude are dimensionless quantities [30].

Table II. Trials with a significantly different, i.e. either significantly higher or significantly lower.

\begin{tabular}{|c|c|c|c|}
\hline Factor & Trial & Session & $\begin{array}{c}\text { Number of } \\
\text { physiological reactions } \\
\end{array}$ \\
\hline \multirow[t]{4}{*}{ Presentation modality } & Visual & 1 & $5^{*}$ \\
\hline & & 2 & 17 \\
\hline & Audiovisual & 1 & $24 *$ \\
\hline & & 2 & 13 \\
\hline \multirow[t]{8}{*}{ Familiarity with stimulus } & All content in familiar tone & 1 & 21 \\
\hline & & 2 & $6^{*}$ \\
\hline & All content in unfamiliar tone & 1 & 24 \\
\hline & & 2 & 22 \\
\hline & Familiar content in familiar tone & 1 & 23 \\
\hline & & 2 & 26 \\
\hline & Familiar content in unfamiliar tone & 1 & 24 \\
\hline & & 2 & $35^{*}$ \\
\hline \multirow[t]{3}{*}{ Colour } & Red-blue-green-yellow-black & 1 & 22 \\
\hline & & 2 & 23 \\
\hline & & 3 & $29^{*}$ \\
\hline \multirow[t]{3}{*}{ Startle } & Startling sound & 1 & $3^{*}$ \\
\hline & & 2 & 22 \\
\hline & & 3 & 16 \\
\hline \multirow[t]{2}{*}{ Anticipation } & Anticipated aural pattern & 1 & 17 \\
\hline & & 2 & $18^{*}$ \\
\hline \multirow[t]{2}{*}{ Association } & Associative aural pattern & 1 & 19 \\
\hline & & 2 & 6 \\
\hline
\end{tabular}

*Number of physiological reactions from that observed in the baseline condition, using $\chi^{2}, p<0.05$.

(audiovisual trial, session 1 in Table II), for which the total number of physiological reactions was significantly different from baseline $\left(\chi^{2}=3.93, p=0.0476\right)$. This finding echoed the perspective of the parent, who predicted considerable reaction to the audiovisual stimulus.

There were also cases where the number of reactions derived from the physiological signals contradicted the parent's prediction. Of particular interest are the factors for which Sean's mother expected intense reactions, but in fact elicited no significant change in Sean's physiological signals. One example is the case of test 2(b) in Table I. In this trial, Sean listened to a random audio sequence consisting of words that were very unfamiliar to him (i.e. names of different planets in the solar system), and one familiar word (i.e. his name). The unfamiliar words were announced in a stranger's voice, while the familiar word was presented in his mother's 
Table III. Proportion of reactions in each physiological signal. Each row shows the proportion of the total number of reactions in the given trial occurring in each physiological modality.

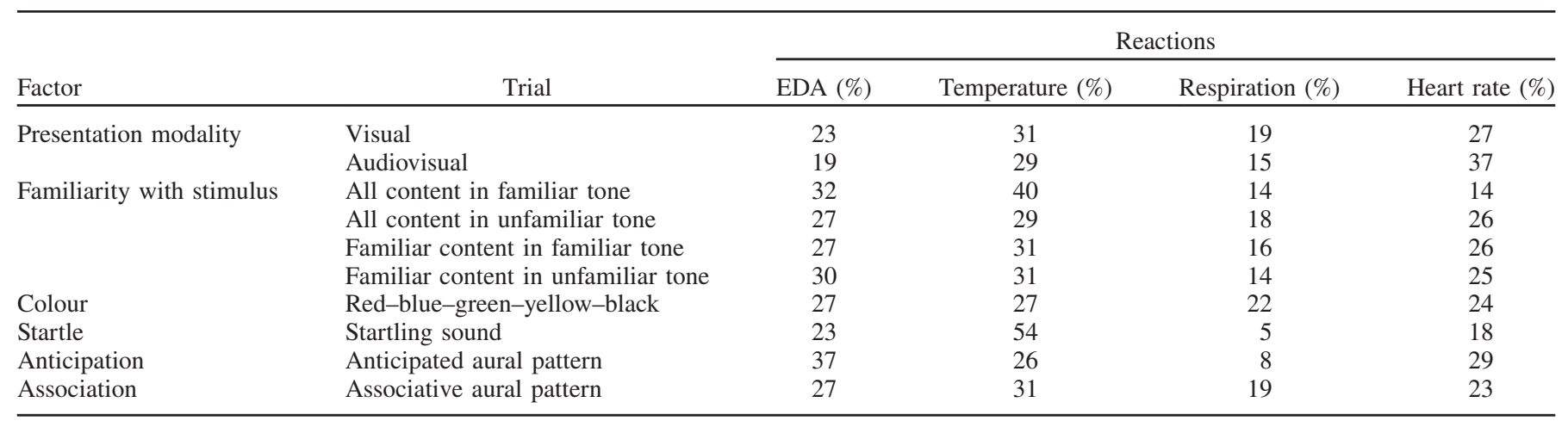
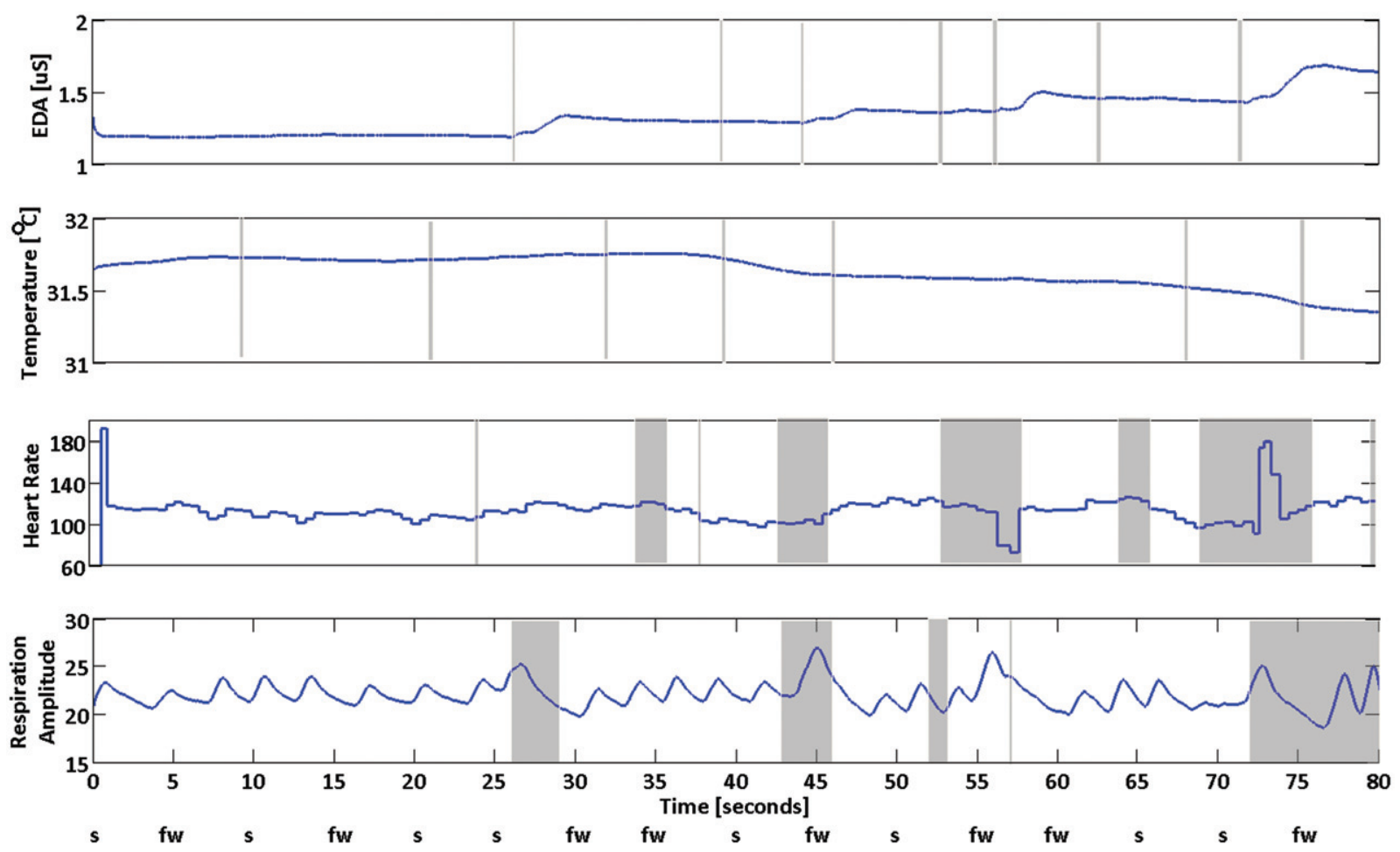

Figure 3. Client's physiological reactions to audiovisual stimulus as detected by the algorithm. The sequence of letters at the bottom of the graph identifies the type of stimulus: 's' denotes a static gray image, and 'fw' denotes a fireworks video. Vertical lines denote reactions in the EDA and skin temperature plots, while shaded blocks denote reactions in the respiration and heart rate signals, as detected by the algorithm. In this trial, the total number of reactions in the client's physiological signals $N=24$ was significantly different from the corresponding sessional baseline $\left(\chi^{2}=3.93\right.$, $p=0.0476)$.

voice. Sean's mother strongly believed that her son's reaction would be most significant in the latter case, as both the content and the tone of the keyword were very familiar to him. However, the physiological results proved the opposite. While there were scattered reactions to the familiar stimulus, no consistent reaction to the familiar stimulus was detected. We repeated this test in another session, and observed similar disagreement between the parent's prediction and physiological findings. This can be seen from Table II, under the third trial of the second factor (i.e. factor: familiarity with stimulus, trial: familiar content in familiar tone). For this type of trial, the total number of physiological reactions showed no significant change from baseline in both sessions.

Another case of strong disagreement between the parent's prediction and physiological reactions is seen in the paper crumple sound startle test (test 4(a) in Table I). While Sean's mother predicted a definite reaction to the paper crumple sound, Sean's physiological signals exhibited very relaxed patterns, which resembled those of baseline. This is reflected in Table II. The total number of reactions to the startling sound was significantly lower than baseline in the first session, and showed no significant difference from baseline in the second and third sessions.

These results suggest that the caregiver's understanding of the client's reactions to sensory and perceptual stimuli may be incomplete. Physiological data may complement caregiver acumen in deciphering the reactions of non-verbal clients with severe and multiple disabilities.

In the long run, if a client is trained sufficiently to realize that his feelings or choices can be conveyed through his body signals, then he/she may perceive a sense of control over the outcome of different situations. Furthermore, drawing on 
evidence from biofeedback studies [31, 32], the client can potentially be trained to voluntarily control his/her peripheral autonomic signals and thus attain an independent voice. This may avert the cognitive, motivational, and affective deficits associated with learned helplessness [33, 34].

\section{Limitations}

A physiological signal may change as a result of any number of internal or external stimuli. For example, EDRs may arise from spontaneous increases in EDA, internal stimulation such as mental activity, and external stimulation such as startling noises [35-37]. The task of separating out the source of these changes is non-trivial and has yet to be successfully addressed. In this study, we implicitly assumed that all physiological changes were due to unconscious reactions to the presented stimuli, running the risk of reporting inflated reaction counts. To mitigate this risk, we have compared reaction counts to each stimulus against baseline activity, assuming that the non-stimuli induced reactions occur equally in all recordings.

While our results suggest potential value in physiological signals, reaction count analysis alone is unlikely to tell the complete story about Sean's feelings and preferences. Patternbased analyses, a broader range of affective stimuli, additional physiological signals, a greater number of test sessions and replication with a larger population are required to gain a fuller appreciation of the client preferences. Furthermore, the method in this study did not give us any information about valence, only arousal.

\section{Conclusion}

We explored the potential of identifying arousing stimuli for a non-verbal client through analysis of his physiological signals. We followed an exploratory approach through which the effect of six different groups of stimuli on the client's electrodermal, thermal, respiration, and heart rate reactions was investigated. We found that the client was more aroused by audiovisual stimuli, aural stimuli articulated in unfamiliar voice, and aural stimuli containing anticipatory patterns. These findings did not agree with the prediction of client's mother about her son's reaction to these stimuli, implying that physiological data may complement caregiver acumen in deciphering the reactions of non-verbal clients with severe and multiple disabilities. We also found that temperature was the most labile physiological signal of the client in this case study.

\section{Declaration of interest}

The authors would like to acknowledge funding for this work from the Natural Sciences and Engineering Research Council of Canada (NSERC), the Canada Research Chairs Program, the WB Foundation, the Remad Foundation and Bell Canada.

\section{References}

1. Blain S, Chau T, Mihailidis A. Peripheral signals as access pathways for individuals with severe disabilities: A literature appraisal. The Open Rehabilitation Journal 2008;1:27-37.
2. Craig A, Tran Y, McIsaac P, Boord P. The efficacy and benefits of environmental control systems for the severely disabled. Medical Science Monitor 2005;11(1):RA32-RA39.

3. Tai K, Blain S, Chau T. A review of emerging access technologies for individuals with severe motor impairments. Assistive Technology 2008;20:204-219.

4. Tsukahara R, Aoki H. Skin potential in letter recognition task as an alternative communication channel for individuals with severe motor disability. Clinical Neurophysiology 2002;113:1723-1733.

5. Holand S, Arlette G, Laude D, Meyer-Bisch C, Elghozi J. Effects of an auditory startle stimulus on blood pressure and heart rate in humans. Journal of Hypertension 1999;17(Suppl 12):1893-1897.

6. Gillespie W. The effects of embedded low intensity aural and visual stimuli of neutral and affective content on skin conductance response. Dissertation Abstracts International 1987;48(4-A): 778-779.

7. Hart JD. Physiological responses on anxious and normal subjects to simple signal and non-signal auditory stimuli. Psychophysiology 1974;11(4):443-451.

8. Carol D. Signal value and physiological response to affective visual stimuli (physiological response to affective visual stimuli, observing signal value change effects on forehead pulse amplitude and galvanic skin response. Psychonomic Science 1971;25:94-96.

9. Dindo L, Fowles DC. The skin conductance orienting response to semantic stimuli: Significance can be independent of arousal. Psychophysiology 2008;45:111-118.

10. Kistler A, Mariauzouls C, Von Berlepsch K. Fingertip temperature as an indicator for sympathetic response. International Journal of Psychophysiology 1998;29:35-41.

11. Castaneda JO, Segerstrom SC. Effects of stimulus type and worry on physiological response to fear. Anxiety Disorders 2004;18: 809-823.

12. Kim KH, Bang SW, Kim SR. Emotion recognition system using short-term monitoring of physiological signals. Medical \& Biological Engineering \& Computing 2004;42:419-427.

13. Blain S, Mihailidis A, Chau T. Assessing the potential of electrodermal activity as an alternative access pathway. Medical Engineering and Physics 2008;30(4):498-505.

14. Tomaka J, Blascovich J, Swart L. Effects of vocalization on cardio-vascular and electrodermal responses during mental arithmetic. International Journal of Psychophysiology 1994;18(1): 23-33.

15. Blain S. Physiologically-mediated interaction between children with profound disabilities and their environment [dissertation]. Toronto (ON): University of Toronto; 2010, 188 p. Available from: https://tspace.library.utoronto.ca/bitstream/1807/32964/1/ Blain_Stefanie_L 201011_PhD thesis.pdf

16. Crawford MR, Schuster JW. Using microswitches to teach toy use. Journal of Developmental and Physical Disabilities 1993;5: 349-368.

17. Lancioni GE, Lems S. Using a microswitch for vocalization responses with persons with multiple disabilities. Disability and Rehabilitation 2001;23:745-748.

18. Lancioni GE, Singh NN, O'Reilly MF, Sigafoos J, Didden R, Oliva D, Calzolari C, Montironi G. A learning setup for a postcoma adolescent with profound multiple disabilities involving small forehead movements and new microswitch technology. Disability and Rehabilitation: Assistive Technology 2007;2:293-297.

19. Lancioni GE, Singh NN, O'Reilly MF, Sigafoos J, Didden R, Smaldone A, Oliva D. Helping a man with multiple disabilities increase object-contact responses and reduce hand stereotypy via a microswitch cluster program. Journal of Intellectual \& Developmental Disability 2008;33(4):349-353.

20. Vetrugno R, Liguori R, Cortelli P, Montagna P. Sympathetic skin response: Basic mechanisms and clinical applications. Clinical Autonomic Research 2003;13(4):256-270.

21. Boucsein W. Electrodermal activity. New York: Plenum Press; 1992. $442 \mathrm{p}$

22. Toyokura M. Waveform and habituation of sympathetic skin response. Electroencephalography and clinical Neurophysiology 1998;109:178-183.

23. Johansen K, Burggren WW, editors. Skin arteriovenous anastomoses, their control and role in thermoregulation: Cardiovascular shunts. Alfred Benzon Symposium. Vol. 21. Copenhagen: Musnksgaard; 1985. pp 433-448. 
24. Elam M, Wallin BG. Skin blood flow responses to mental stress in man depend on body temperature. Acta Physiology Scandinavica 1987;129:489-497.

25. Lacey B, Lacey J. Cognitive modulation of time-dependent primary brachycardia. Psychophysiology 1980;17(3):209-221.

26. Steptoe A, Fieldman G, Evans O, Perry L. Cardiovascular risk and responsivity to mental stress: The influence of age, gender and risk factors. Journal of Cardiovascular Risk 1996;3(1):83-93.

27. Gomez P, Shafy S, Danuser B. Respiration, metabolic balance and attention in affective picture processing. Biological Psychology 2008;78(2):138-149.

28. Jonghwa K, Andre E. Emotion recognition based on physiological changes in music listening. IEEE Transactions on Pattern Analysis and Machine Intelligence 2008;30(12):2067-2083.

29. Agresti A. Categorical data analysis, 2nd ed. Hoboken, NJ: John Wiley \& Sons; 2002. 744 p.

30. Freedman RR, Ianni P. Self-control of digital temperature: Physiological factors and transfer effects. Psychophysiology 1983; 20:682-689.
31. Nestoriuc Y, Martin A. Efficacy of biofeedback for migraine: A meta-analysis. Pain 2007;128(1-2):111-127.

32. McGuinness S. Learned helplessness in the multiple sclerosis population. Journal of Neuroscience Nursing 1996;28(3):163-170.

33. Nolen-Hoeksema S, Girgus JS, Seligman MEP. Learned helplessness in children: A longitudinal study of depression, achievement, and explanatory style. Journal of Personality and Social Psychology 1986;51(2):435-442.

34. Ben-Shakhar G, Dolev K. Psychophysiological detection through the guilty knowledge technique: Effects of mental countermeasures. Journal of Applied Psychology 1996;81:273-281.

35. Hay JE, Taylor PK, Nukada H. Auditory and inspiratory gaspevoked sympathetic skin response: Age effects. Journal of Neurological Sciences 1997;148:19-23.

36. Weisz J, Czigler I. Age and novelty: Event-related brain potentials and autonomic activity. Psychophysiological 2006;43:261-271.

37. TTLAPI Programmer's Reference Manual. Covers TTLLiveCtrl 2.1.1698+, TTLSessionMgrCtrl 2.0.421, Revision 15, July 11, 2005. 